\title{
ISOCAM mid-infrared spectroscopy and NIR photometry of the HII complex N4 in the Large Magellanic Cloud ${ }^{\star}$
}

\author{
A. Contursi ${ }^{1}$, M. Rubio ${ }^{2}$, M. Sauvage ${ }^{3}$, D. Cesarsky ${ }^{1,5}$ R. Barba ${ }^{4}$, and F. Boulanger ${ }^{5}$ \\ 1 Max-Planck-Institut für Extraterrestrische Physik (MPE), Postfach 1312, 85741 Garching, Germany \\ e-mail: contursi@mpe.mpg.de \\ 2 Departamento de Astronomía Universidad de Chile, Casilla 36-D, Santiago, Chile \\ 3 CEA/DSM/DAPNIA/Service d'Astrophysique, CE Saclay, 91191 Gif-sur-Yvette Cedex, France \\ 4 Departamento de Física, Universidad de La Serena, Chile \\ 5 Institut d'Astrophysique Spatiale, Bat. 121, Université Paris XI, 91450 Orsay Cedex, France
}

Received 18 October 2006 / Accepted 16 January 2007

\section{ABSTRACT}

\begin{abstract}
Context. We present the analysis of ISOCAM-CVF and the $J, H$, and $K_{\mathrm{s}}$ photometry data for the HII region complex N4 in the Large Magellanic Cloud (LMC).

Aims. The aim is twofold: 1) to study the connection between the interstellar medium and the star content of this region; 2) to investigate the effects of the lower-than-Galactic metallicity on dust properties.

Methods. A dust-features - gas lines - continuum fitting technique on the whole ISOCAM-CVF data cube allows the production of images in each single emission and detailed analysis of dust (both continuum and bands), and ionized gas. The near infrared (NIR) photometry provides, for the first time, information on the stellar content of N4.

Results. The mid-infrared (MIR) spectral characteristics of N4 are those expected for an HII region complex, i.e. very similar to those observed in galactic HII regions. The images in single dust feature bands and gas lines clearly show that the HII region core is completely devoid of the carriers responsible for the aromatic features (AFs). On the other hand, the ionized gas arises almost completely in this dust cavity, where the two main exciting stars of N4 are also located. The HII region models from Stasińska (1982, A\&AS, 48, 2999) predict an HII region size that corresponds to the observed size of the dust cavity.

We find evidence that the effect of lower-than-Galactic metallicity (although not as extreme as in the case of LMC) on the carriers responsible for the AFs is not to prevent their formation or to modify their chemical properties, but to enhance their destruction by the high and hard interstellar radiation field. We argue that this is the dominant process responsible for the absence of AFs in the HII region core. We show that this mechanism is more efficient on smaller dust particles/molecules thus affecting the dust-size distribution. We argue that effects on dust-size distribution, rather than the different dust properties due to a lower metallicity, should be taken into account when analyzing more distant, relatively low-metallicity galaxies.

Finally, the analysis of the stellar content of N4 reveals 7 stars: 4 reddened O MS stars and three stars with envelopes. In particular, one of these seems to be an ultra compact HII region containing an embedded young stellar object.
\end{abstract}

Key words. HII regions - ISM: lines and bands - ISM: structure

\section{Introduction}

N4 (Henize 1956) is an HII complex in the northwest part of the Large Magellanic Cloud (LMC). The physical characteristics of the ionized gas and associated molecular cloud, have been extensively studied by Heydari-Malayeri and Lecavelier des Etangs (1994) through optical and submillimeter line spectroscopy.

The $\mathrm{H} \alpha$ emission of N4 (Fig. 1, kindly provided by HeydariMalayeri) clearly shows that this HII complex is composed of two ionized nebulae: N4A and N4B. In this paper we will concentrate only on $\mathrm{N} 4 \mathrm{~A}$, which is brighter and younger than N4B and is composed of a bright dense front to its northeastern part and a more diffuse low-surface brightness (partially density bounded) component in its southern part. The main ionizing sources in N4A are two stars not visible at optical wavelengths (marked in Fig. 1 as A and B). Heydari-Malayeri \& Lecavelier des Etangs (1994) calculated that the main

* Based on observations with ISO, an ESA project with instruments funded by ESA member states (especially the PI countries: France, Germany, the Netherlands and the United Kingdom) and with the participation of ISAS and NASA. ionizing sources correspond to either one $60 M_{\odot}$ star or two $40 M_{\odot}$ stars.

The average extinction in N4 derived from optical gas emission is quite low, $A_{\mathrm{V}}=0.4$ although it is presumably higher in the northeastern front where the gas density reaches its maximum. The parental molecular cloud is formed by two spatial components: one peaking on $\mathrm{N} 4 \mathrm{~A}$ and the other peaking to the east of the HII region. The molecular cloud has two velocity components: a high- velocity, optically-thick red component and a thin blue molecular sheet. This sheet is probably pushed towards us by the HII region pressure (Heydari-Malayeri \& Lecavelier des Etangs 1994).

A complementary study of the hot dust emission in N4A has been done by Contursi et al. (1998) using broad band ISOCAM filters centered at 7 and $15 \mu \mathrm{m}$. At these wavelengths, the emission is dominated by the Aromatic Features seen in Emission (AFEs) between 3 and $10 \mu \mathrm{m}$ and a continuum steeply rising at wavelengths longer than $\sim 10 \mu \mathrm{m}$. The overall mid-infrared (MIR) morphology in N4A is generally very similar to the CO emission, although the MIR and CO peaks do not coincide exactly. The strongest MIR emission comes from the dense 


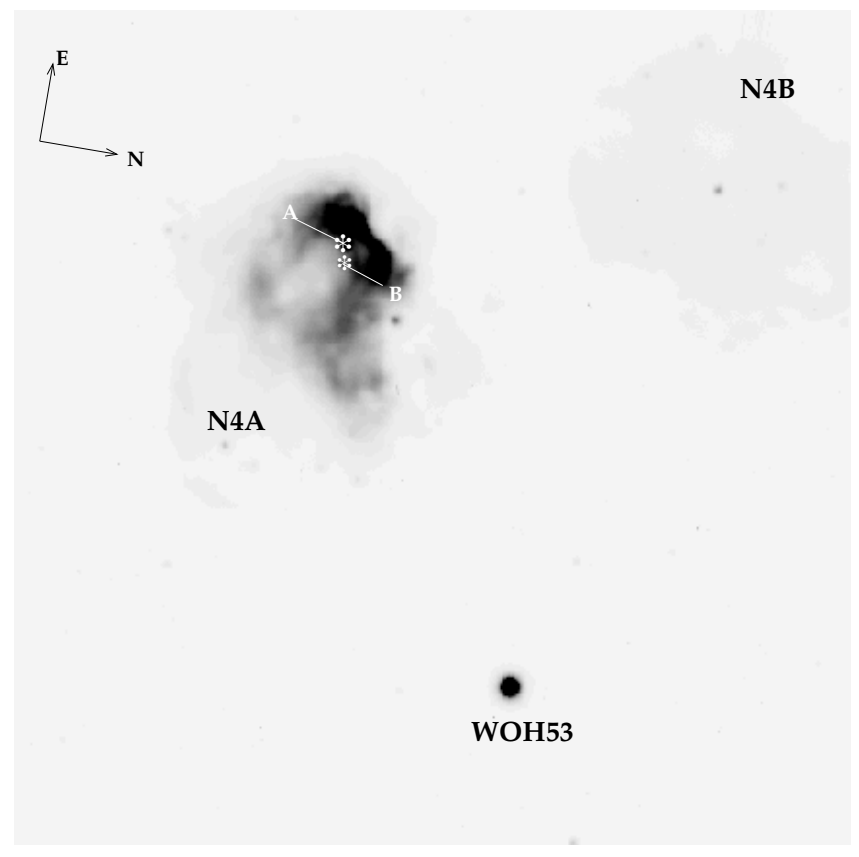

Fig. 1. $\mathrm{H} \alpha$ emission of the LMC HII complex N4A and N4B (from Heydari-Malayeri \& Lecavelier des Etangs 1994). The image has been rotated to the same orientation as the ISOCAM-CVF images.

northeastern front as expected, since here the density of the matter is at its highest. The $15 / 7 \mu \mathrm{m}$ ratio peaks in the region of N4A where the two main exciting stars are supposed to be. Its high value, typical of an HII region, indicates that the emission at $15 \mu \mathrm{m}$ is dominated by a strong continuum and that the emission at $\sim 7 \mu \mathrm{m}$ is significantly reduced. Contursi et al. (1998) suggests that this decrease is due primarily to destruction of the AFEs carriers in hard and strong interstellar radiation fields (ISRF).

This analysis was part of a wide ISOCAM campaign aiming at studying the interstellar medium (ISM) properties in the Magellanic Clouds with particular emphasis on the role played by metallicity, taking full advantage of the unprecedented (at that time) spatial resolution provided by ISO at these wavelengths and to the proximity of the Magellanic Clouds. One of the outcomes of the broad band ISOCAM imaging study of N4 was that this region appears as a classical Galactic HII complex, composed mainly of three phases: 1) the HII region itself close to the exciting stars corresponding to the maximum of the $15 / 7 \mu \mathrm{m}$ ratio; 2) the interface between the HII region and the molecular cloud (photon-dominated region or PDR) where the MIR emission reaches its maximum due to both high dust density and relatively high ISRF; 3) the parental molecular cloud, where the MIR emission is still dominated by AFEs probably arising from the outskirts of the molecular clouds and excited mainly by the general LMC ISRF.

In this paper we present a more detailed study of the MIR emission properties of N4A analyzing a very high signal-tonoise spectro-imaging data set carried out with the circular variable filter (CVF) on ISOCAM. Thanks to the spectral capabilities, we are able to directly show that our previous physical interpretation of the broad band MIR photometry data of N4 were correct. We also present new high resolution $J, H$ and $K_{\mathrm{s}}$ images, taken at Las Campanas Observatory (Chile). All these data allow us to better investigate the existence of detailed metallicity signatures on the dust emission that do not show up in the broad band emission and to relate dust, ionized gas, and stellar content. These data provide high resolution analogs for the study of the ISM in conditions similar to those of young galaxies in the distant Universe that are becoming more and more available with the advent of large ground-based telescopes (VLT, Keck, ALMA) and space missions (SPITZER, HERSCHEL).

\section{The data}

\subsection{ISOCAM-CVF data redution}

A complete scan of the two long-wavelength Circular Variable Filters (CVF) was performed going down in wavelengths, step by step, first from 16.2 to $9.0 \mu \mathrm{m}$ (LW-CVF2) and second from 9.4 to $5.0 \mu \mathrm{m}$ (LW-CVF1). At each CVF step, between 7 and 11 frames of $2.1 \mathrm{~s}$ were taken. The total observing time was $2974 \mathrm{~s}$. The pixel field of view was $6^{\prime \prime}$ and the full field of the observation $3^{\prime} \times 3^{\prime}$ centered on RA $=4: 52: 8.2$ and Dec $=-66: 55: 16(\mathrm{~J} 2000)$. The data reduction was performed as described in Boulanger et al. (2004). The reduced data cube can be retrieved from the ISO archive at the site http://www.iso.vilspa.esa.es as Highly Porcessed Data Products (HPDP) under the name Mid-IR Spectro Imaging ISOCAM CVF Observations.

\subsection{Near infrared broad band filters data}

Near infrared $J, H$, and $K_{\mathrm{s}}$ deep images of N4A were obtained with the Dupont $2.5 \mathrm{~m}$ telescope at Las Campanas Observatory (Chile) in the night of December 27, 1996 with the $256 \times$ 256 NICMOS III camera IRCAM (Persson et al. 1992). The spatial resolution of the system was $0.35^{\prime \prime} / \mathrm{pixel}$, and the typical seeing was $0.9^{\prime \prime}$ in $K_{\mathrm{s}}$.

The observation consisted of a series of 10 frames, each individual frame with an integration time of $20 \mathrm{~s}$ per filter. This procedure was repeated in a 9-position mosaic with a separation of $20^{\prime \prime}$. The total integration time in each filter resulted in $1800 \mathrm{~s}$ in $K_{\mathrm{s}}, 1800 \mathrm{~s}$ in $H$, and $2000 \mathrm{~s}$ in $J$. Sky frames in each filter were taken in a field with faint stars and no extended emission at 12" W and 134" $\mathrm{S}$ of the source position. The sky field was observed in a similar way as for N4. To produce the final images, each image was flat-fielded and sky-subtracted, and then median-averaged-combined using IRAF procedures. The final images were registered with respect to the $K_{\mathrm{s}}$ image by means of several common stars. The resulting mosaic covers a $65^{\prime \prime} \times 76^{\prime \prime}$ area.

Aperture photometry in each filter was performed with IRAF/DAOPHOT on the mosaic. For the purpose of this study we concentrated on those sources brighter than $K_{\mathrm{S}}<13$. The stars associated to N4A are shown in Fig. 5, and Table 1 gives the NIR magnitudes and colors of these stars. The photometric errors are $\sigma_{K_{\mathrm{s}}}=0.06 \mathrm{mag}, \sigma_{H}=0.05$ and $\sigma_{J}=0.03$. Photometry of stars in our mosaic far from the N4A nebulosity were compared to their 2MASS photometry and showed a good agreement. We estimated the flux contribution from the nebulosity to our measured photometry to be less than $10 \%$ and $5 \%$ in the $K_{\mathrm{s}} J$ bands, respectively.

\section{Results}

In this section we describe the MIR spectra characteristics in different regions of $\mathrm{N} 4$ and its morphology in the dust components, i.e. dust-features and dust-continuum, and the gas-lines, and we relate them to the stellar content as revealed by the NIR photometry. 

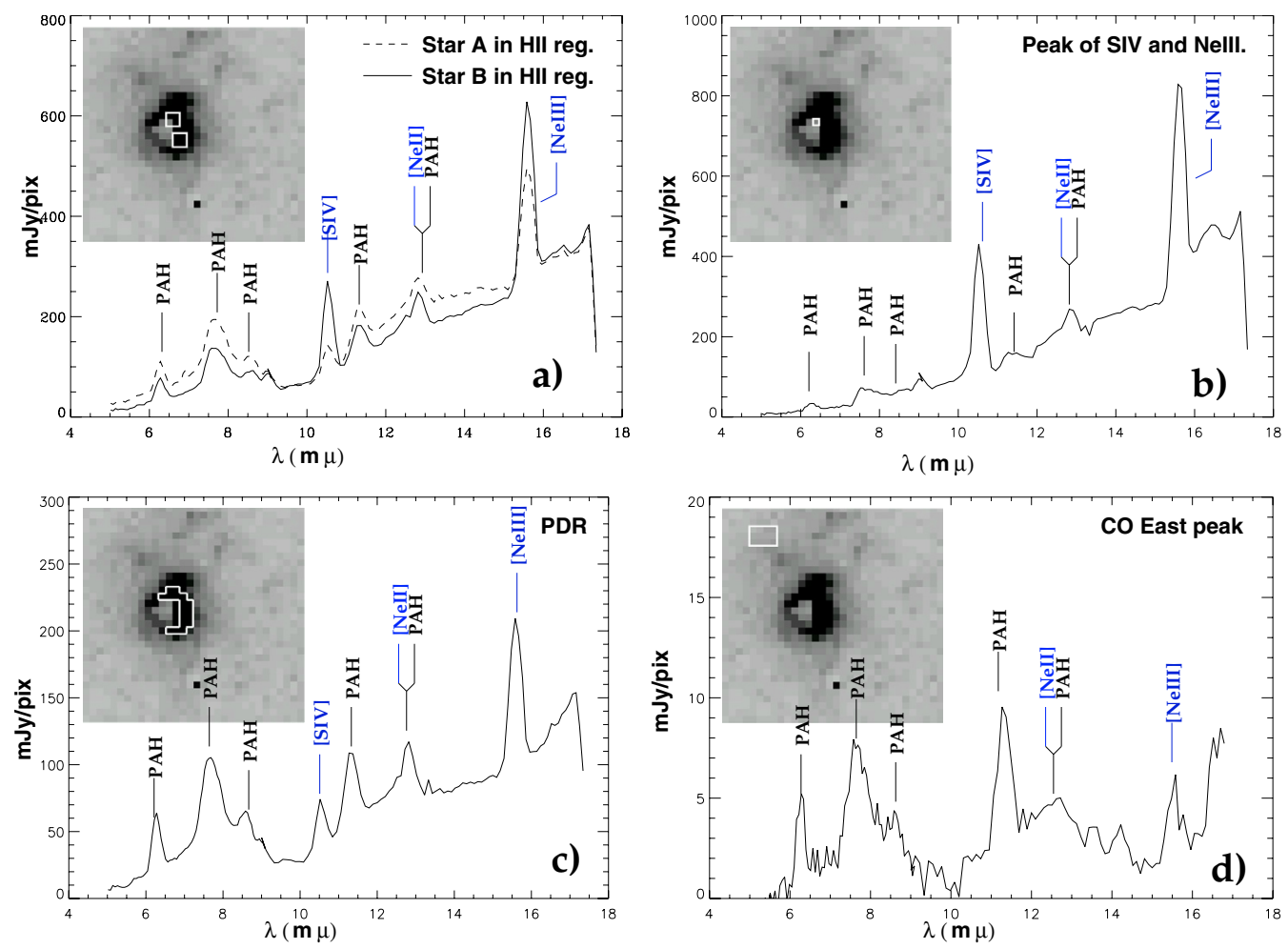

Fig. 2. ISOCAM CVF spectra of four different regions of N4A. The regions where the spectra were extracted are shown in each panel on the gray-scale image in the $7.7 \mu \mathrm{m}$ aromatic feature. Note that the steep rise or decrease at the very end of the spectrum is an instrument artifact and should not be considered as real.

\subsection{The mid-infrared spectrum of $N 4 A$}

Figure 2 shows the MIR spectra of 4 regions in N4A representative of 4 typical components of an HII complex. The upper left panel spectra (a) were produced by averaging 4 pixels $\left(6^{\prime \prime} /\right.$ pix $)$ centered on the position of the two main exciting stars in N4A. The upper right panel (b) shows the spectrum of the ionized gas peak emission. The bottom left spectrum (c) is the average of 18 pixels on the bright northeastern front of N4A; the bottom right spectrum (d) corresponds to the $\mathrm{CO}$ eastern peak, outside the ionized cloud (Contursi et al. 1998).

The spectra around the stars (a) and on the northeastern front (c) are dominated by the AFEs and a continuum longward of $10 \mu \mathrm{m}$ which becomes more and more prominent approaching the stars $(b$ and $a)$. AFEs are stronger on the northeastern front of N4A, as expected in a region where both density and ISRF are high. This spectrum (c) is typical of PDRs, thus confirming that this region is the interface between the HII region and the molecular cloud of N4A.

In the spectra (panel a) corresponding to the regions close to the stars, the prominent features are the fine-structure gas lines of SIV at $10.5 \mu \mathrm{m}$, NeII at $12.8 \mu \mathrm{m}$ (although blended with the AFE at $12.7 \mu \mathrm{m}$ ), and NeIII at $15.6 \mu \mathrm{m}$. The AFEs to continuum ratio reaches its minimum in the region where the fine-structure lines are the most intense (b) and AFEs practically disappear. The spectrum (d) towards the eastern $\mathrm{CO}$ peak presents very weak AFEs and almost no continuum. These are characteristics of quiescent regions, which suggests that the emission arises from a thin external shell of the molecular cloud probably corresponding to its red velocity component.

From this global spectral analysis, it appears that N4 MIR dust and gas properties are very similar to what is found in galactic HII complexes (Cesarsky et al. 1996b,a), i.e. its MIR emission spectrum is typical of a HII/PDR/molecular cloud combination.

\subsection{Emission in the single dust bands and gas lines}

Thanks to the very high signal-to-noise ratio and the fact that we have spatial and spectral information on a region of $3^{\prime} \times 3^{\prime}$ centered on N4A, this data set is perfectly suitable for a detailed spectral and spatial analysis in the single AFEs, gas lines, and pure continuum. We produced images in each dust feature (namely, $6.2 \mu \mathrm{m}, 7.7 \mu \mathrm{m} 8.6 \mu \mathrm{m} 11.3 \mu \mathrm{m}$ ) and fine structure lines (SIV, NeII and NeIII) in the following way. Following Boulanger et al. (1998), we performed a Lorentzian fit on the dust bands and a Gaussian fit on the gas lines, to the whole data cube, producing maps in each dust feature and fine-structure line of the ionized gas. First we fit the $6.2 \mu \mathrm{m}, 7.7 \mu \mathrm{m}$, and $8.6 \mu \mathrm{m}$ bands together and a straight line as a continuum letting the slope vary at each pixel. Then, we fit the SIV, $11.3 \mathrm{NeII}(+12.7 \mu \mathrm{m})$ and NeIII lines together, and a straight line as a continuum. The reason we separately fit the two parts of the spectrum is that in the HII region core the continuum shortward and longward $10 \mu \mathrm{m}$ might not have the same origin and therefore the same slopes (see discussion in Sect. 4.2). We then subtracted the fitted lines and bands from the original data cube, pixel by pixel, producing "pure" continuum spectra. We visually inspected the subtracted data cube to ensure that no "absorption" features were produced due to overestimated lines/features. From the "pure" continuum spectrum, we built images in the ISOCAM LW5 $(6.5-7.0 \mu \mathrm{m})$ and LW9 $(14-16 \mu \mathrm{m})$ filters. They represent the pure continua shortward and longward of $10 \mu \mathrm{m}$, respectively. The NeII $(12.7 \mu \mathrm{m})$ line was fitted with a Gaussian on the whole cube, even though we know that it is blended with the aromatic feature at $12.7 \mu \mathrm{m}$, which should be fitted with a Lorentzian. 


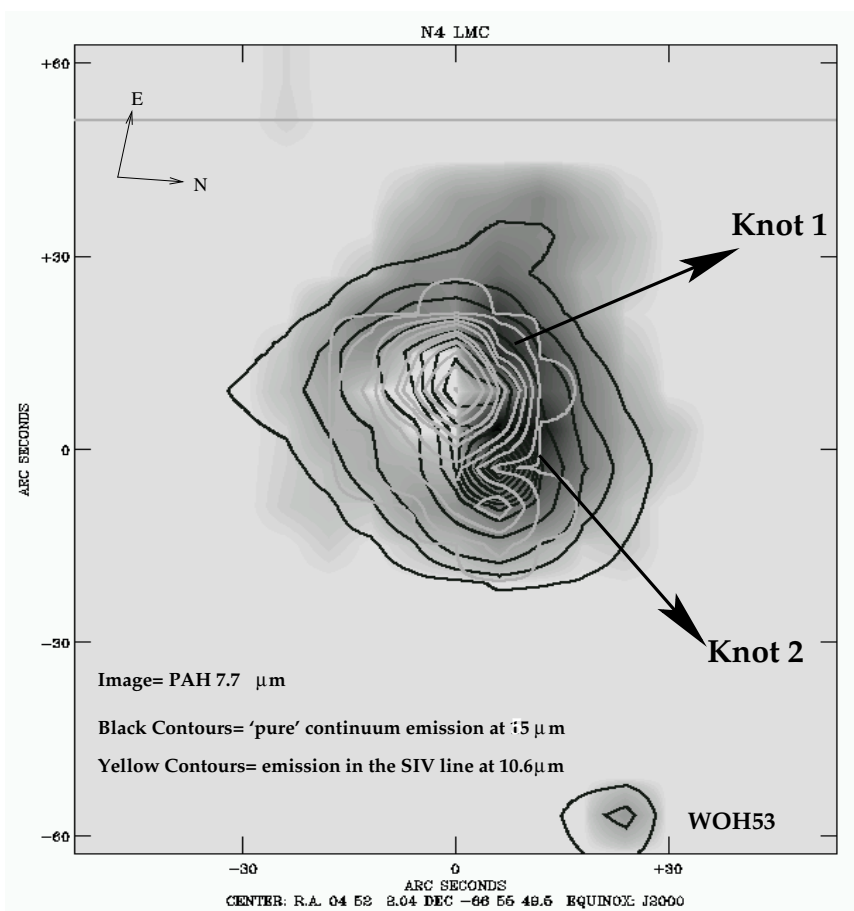

Fig. 3. Image: map from the Lorentzian fit of the $7.7 \mu \mathrm{m}$ dust emission feature. Gray and black contours: from the map of Gaussian fit in the SIV line and the "pure" continuum in the LW9 filter $(15 \mu \mathrm{m})$ emission, respectively.

Therefore the NeII image also contains the $12.7 \mu \mathrm{m}$ AFE. The resulting images are shown in Figs. 3 and 4. All emission shown hereafter are clipped at $3 \sigma$.

Figure $3^{1}$ shows the $7.7 \mu \mathrm{m}$ emission obtained through Lorentzian fits. Contours represent the SIV line emission obtained by applying Gaussian fits and the "pure" $15 \mu \mathrm{m}$ (LW9) continuum emission. In this image it is clear that the AFE is coming from a shell surrounding the HII region core and that this shell is particular bright in the PDR (i.e. on the northeastern front). There are 2 bright compact knots on this shell (numbered in Fig. 3), not resolved at the $7.7 \mu$ m resolution ( $\left.\sim 7^{\prime \prime}\right)$. Most of the ionized gas emission arises from the cavity formed by the dust shell, and it seems centered on a point-like source. A similar result has been published recently by Zavagno et al. (2006) on the massive galactic star-forming region RCW 79. The $15 \mu \mathrm{m}$ pure continuum emission peaks close to knot\#2 in the PDR dust shell and has a weaker emission in the $7.7 \mu \mathrm{m}$ cavity where SIV peaks. Figure 4 shows the $7.7 \mu \mathrm{m}$ image with the NeIII contours. The general picture is very similar to what is shown in Fig. 3: the ionized gas traced by the NeIII emission is mostly contained in the dust cavity and does not show a secondary peak corresponding to the secondary peak seen in the SIV line emission, although this can be due in part to the poorer resolution at $15.6 \mu \mathrm{m}$ than at $10.5 \mu \mathrm{m}$. The main difference between the two ionized gas lines emission is that the NeIII emission is much more extended than the SIV emission towards the southern part of the nebula. This is also the side where the HII region is partially bounded (HeydariMalayeri \& Lecavelier des Etangs 1994) and where the gas can expand.

\footnotetext{
1 Maps obtained from the CVF data have not been rotated north-south because, at these wavelengths, the images are undersampled and a rotation would conserve neither the flux nor the spatial distribution. To make the comparison among data at other wavelengths easier, we have rotated the $\mathrm{H} \alpha$ and the NIR images to the same ISOCAM orientation.
}

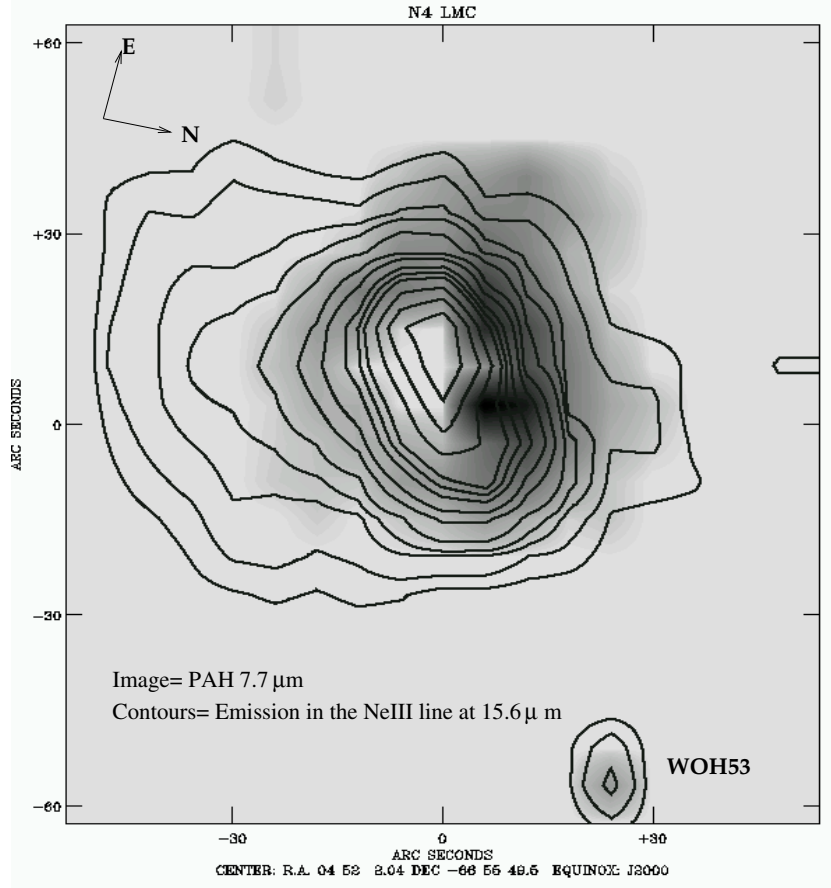

Fig. 4. Image: map from Lorentzian fit of the $7.7 \mu \mathrm{m}$ dust emission feature. Contours: from the map of Gaussian fit in the NeIII line.

All other images in the other dust bands (not shown here) are very similar to the $7.7 \mu \mathrm{m}$ emission. The $\mathrm{NeII}(+12.7 \mu \mathrm{m})$ distribution is also very similar to the distribution of the other AFEs everywhere but in the HII region core where there is a peak. This suggests, as expected, an increasing contribution by the NeII line to the $12.7 \mu \mathrm{m}$ feature when approaching the exciting stars. We will come back to the relative contribution of the NeII line and the $12.7 \mu \mathrm{m}$ AFE in Sect. 4.1.

\section{3. $J, H$ and $K_{\mathrm{s}}$ photometry}

The $J-H-K_{\mathrm{s}}$ composite image of N4A (Fig. 5) shows an extended and diffuse emission whose morphology is very similar to the shape of the $\mathrm{H} \alpha$ emission seen in Fig. 1 and with NIR color quite uniform across the nebula. Seven stars are visible (numbered in Fig. 5). Stars \#1 and \#3 are the bluest, while the others are much redder. Star \#4, to the north-west of the nebula, is bright and red. Star \#2 is located on the border north of the nebula and shows diffuse emission around it. Photometry of all the seven stars is listed in Table 1 ordered by $J$ magnitudes. Star numbers refer to Fig. 5. Star \#1 and \#3 are associated with stars A and B in Fig. 1.

The comparison with the position of the stars indicated by Heydari-Malayeri \& Lecavelier des Etangs (1994) as the two main exciting stars (marked as A and B in Fig. 1) suggests that they correspond to stars \#1 and \#3 in Fig. 5. This association, however, is not certain because of the lack of astrometry in their data (their Fig. 2). We believe that these are the exciting stars, because if one associates the $\mathrm{H} \alpha$ knot visible in their figure at about $\left(0^{\prime \prime},-27^{\prime \prime}\right)$ from the center of their field with our star \#7, the position of the two exciting stars as indicated in their paper corresponds to the position of stars \#1 and \#3.

The comparison between the $K_{\mathrm{s}}$ band and the $15 \mu \mathrm{m}$ "pure" continuum emission (shown in Fig. 6) clearly indicates that star \#4 corresponds to the strongest peak of the pure continuum emission at $15 \mu \mathrm{m}$. Figure 7 shows the $K_{\mathrm{s}}$ band emission contours on 


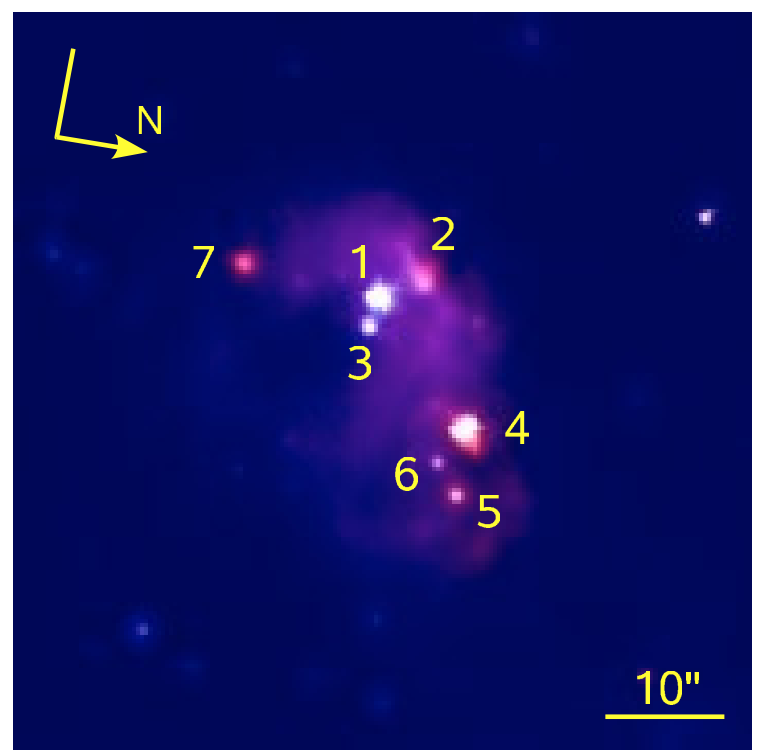

Fig. 5. NIR Composite map oriented like the ISOCAM images: red is $K_{\mathrm{s}}$, green is $H$ and blue is $J$. Point sources identified as stars are numbered as in Table 1 .

Table 1. Observed colors of the 7 stars stars visible in NIR images.

\begin{tabular}{cccc}
\hline \hline Star Nb. & $J_{\text {mag }}$ & $(J-H)$ & $\left(H-K_{\mathrm{s}}\right)$ \\
\hline Star 1(A) & 13.63 & 0.12 & 0.24 \\
Star 2 & 14.16 & 0.32 & 0.97 \\
Star 3(B) & 14.34 & 0.07 & 0.12 \\
Star 4 & 14.50 & 1.01 & 1.26 \\
Star 5 & 16.25 & 0.93 & 0.67 \\
Star 6 & 16.26 & 1.01 & 0.71 \\
Star 7 & 16.35 & 1.04 & 1.60 \\
\hline
\end{tabular}

the SIV line image. The SIV emission arises almost entirely in the region where the 2 bluest stars are located. Finally, Fig. 8 shows that one of the AFE peaks (knot \#2 in Fig. 3 ) is close but does not totally correspond to the bright continuum source (star \#4) and that knot \#1 is located north of the blue stars, very close to where the red elongated feature is visible in the NIR composite map (associated with \#2 in Fig. 5). This suggests that this source and star \#4 are embedded in a high-density dusty region.

Note, however, that the uncertainty on ISO-CVF data astrometry $\left(\leq 6^{\prime \prime}\right)$, plus the fact that the images at different wavelengths are slightly displaced with respect to each other (by up to 1 pixel over the whole ISOCAM spectral range), prevents us from using the ISOCAM astrometry to superpose images at different wavelengths. We align the CVF images with respect to each other and the CVF images on the $K_{\mathrm{s}}$ band image by matching the peak emission of the WOH53 star (marked in Fig. 1).

From the comparison between the ISOCAM and NIR data, we conclude that SIV and NeIII emission, i.e. the ionized gas, is mostly associated with the bluest stars in the cavity of the dust shell. The $15 \mu \mathrm{m}$ continuum peak is mostly associated with the red star \#4 very close but not coincident to one of the peaks of the $7.7 \mu$ m emission (knot \#2). Object \#2 corresponds to knot \#1 in the $7.7 \mu \mathrm{m}$ emission.

\section{Discussion}

In this section we derive some physical conditions of N4 and we compare them with those found in normal metallicity

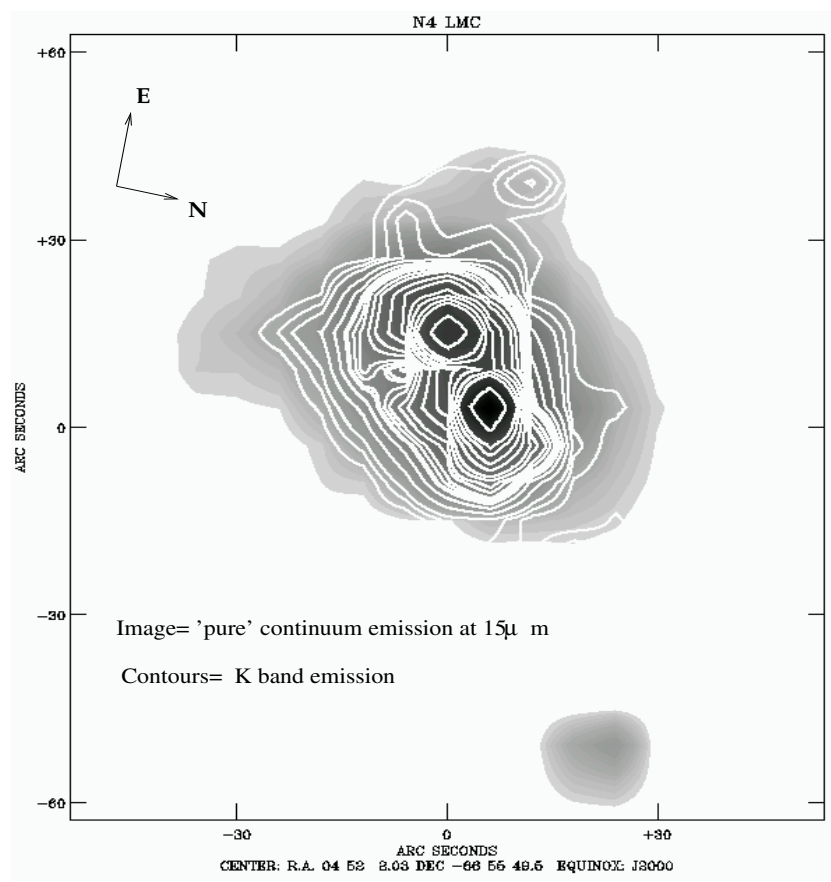

Fig. 6. Image: LW9 $(15 \mu \mathrm{m})$ pure continuum emission; Contours $=K$ band.

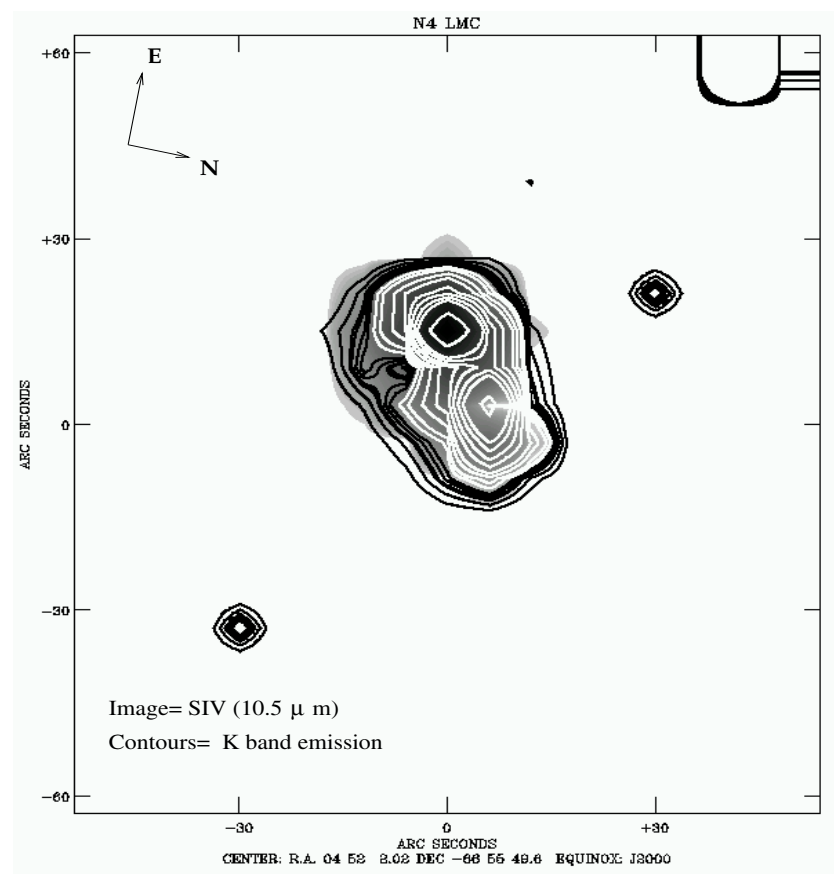

Fig. 7. Image: SIV at $10.5 \mu \mathrm{m}$ from the Gaussian fit; Contours $=K$ band. Contours are shown in two colors (black and white) for the purpose of visualization.

environments. In particular, we use the ionized gas properties to derive the size of the HII region and we compare it to the dustfeature cavity presented in the previous section. Then we analyze the dust emission in detail: we investigate the origin of the MIR continuum and the mechanism responsible for the lack of AFEs in the HII region core. We then compare in detail the dustfeature intensity ratios and widths with those typical of regions in normal metallicity environments. 


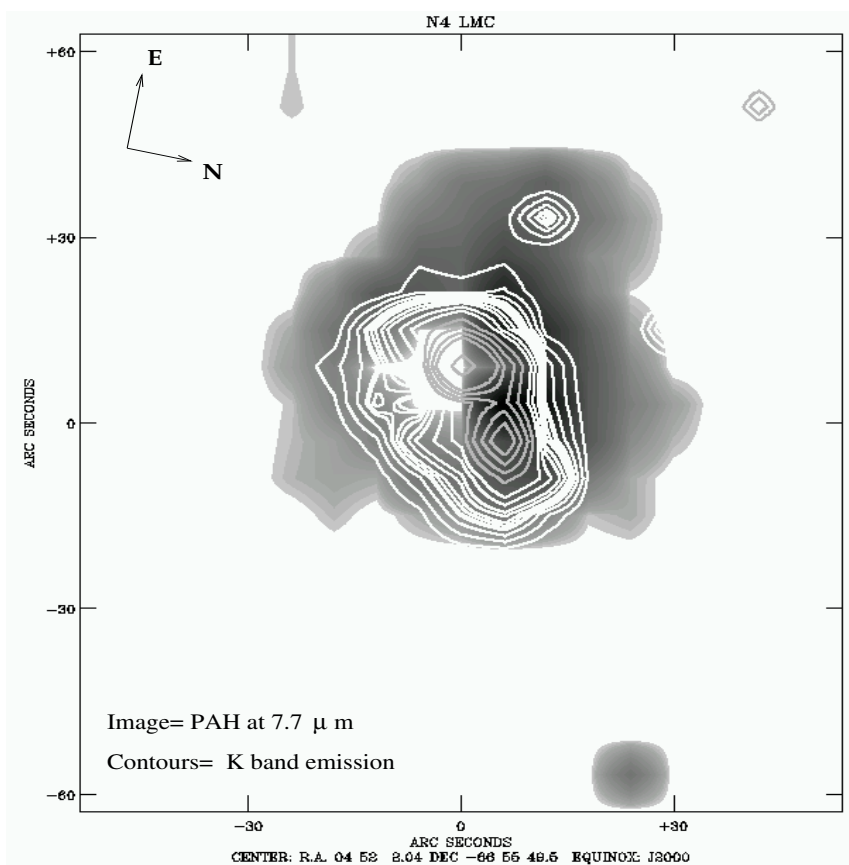

Fig. 8. Image: $\mathrm{PAH}, 7.7 \mu \mathrm{m}$ feature from the Lorentzian fit; Contours $=K$ band.

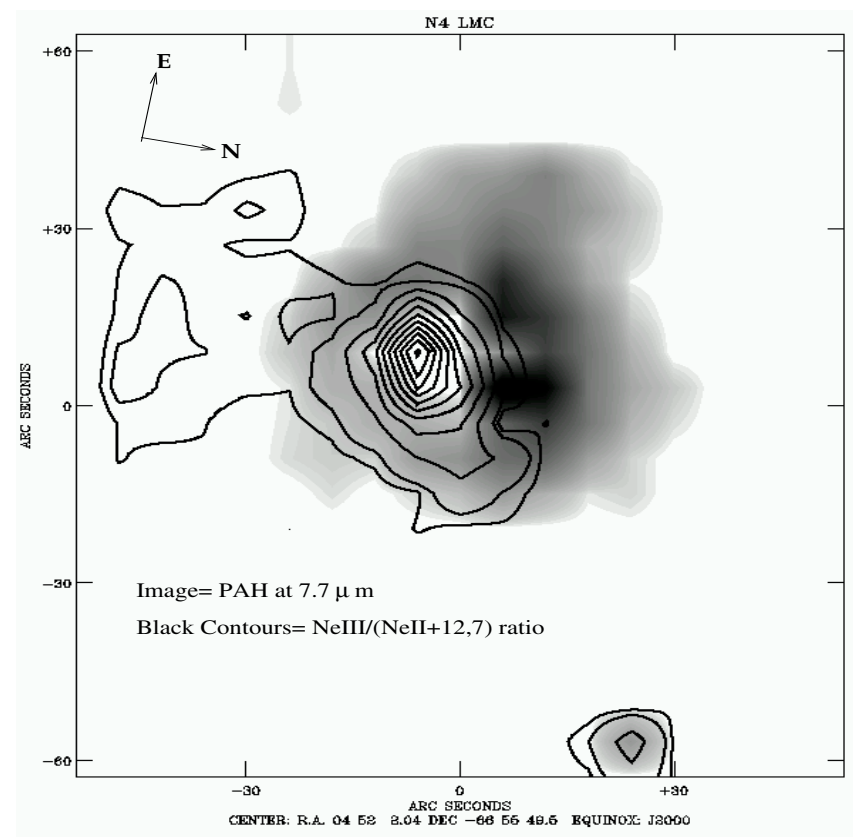

Fig. 9. Image: $\mathrm{PAH}, 7.7 \mu \mathrm{m}$ feature from Lorentzian fit; Contours $=\mathrm{NeIII} / \mathrm{NeII}(+12.7)$ ratio. Contour values are: $0.7,1$, $2,3,4,5,6,7,8,9,10.5$.

\subsection{The ionized gas}

Figure 9 shows the NeIII/NeII ratio contours over the $7.7 \mu \mathrm{m}$ map. As explained above, this ratio has to be considered a lower limit, because AFE at $12.7 \mu \mathrm{m}$ and the NeII at $12.8 \mu \mathrm{m}$ are blended at the ISO spectral resolution. In general, the infrared NeIII/NeII ratio, practically not affected by extinction (Thornley et al. 2000, and references therein), is sensitive to the hardness of the UV radiation field. Thornley et al. (2000) studied how this ratio changes in a sample of star-forming regions belonging to starburst galaxies and find that, after excluding the two systems (IZw 40 and NGC5253) in their sample with low metallicity, the NeIII/NeII ranges from 0.05 to 1 . In a comparable range of NeIII/NeII ratios, Brandl et al. (2006) show that in a sample of starburst galaxies the equivalent width (EW) of the $7.7 \mu \mathrm{m}$ feature does not vary significantly. At higher $(>1) \mathrm{NeIII} / \mathrm{NeII}$ ratios, a weak anti-correlation has been found by Wu et al. (2006) in a sample of blue compact dwarf galaxies, i.e. low metallicity systems.

In $\mathrm{N} 4$ the $\mathrm{NeIII} / \mathrm{NeII}$ ratio peaks in the HII region core, right on top of the bluest stars, with a value equal to $\sim 10$ that is comparable to what has been found in IZw 40 and in 30Dor (Thornley et al. 2000) and in other low metallicity galaxies (Wu et al. 2006; Madden et al. 2006). However, the global ratio is $\gtrsim 0.9$, comparable to the typical value of the normal metallicity extragalactic HII regions. It is also noteworthy that there is an extended emission in NeIII/NeII $(+12.7 \mu \mathrm{m})$ ratio map in the southern part of the HII region.

Table 2 shows the total flux of the fine structure lines SIV, $\operatorname{NeII}(+12.7 \mu \mathrm{m})$, and NeIII. We checked whether the emission in the ionized gas lines of N4A agrees with the prediction of HII region models. We did not perform detailed calculation with models such as CLOUDY (Ferland et al. 1998) or similar, because we have only 3 lines available, one of which is an upper limit. We instead compared our results with the model prediction from Stasińska (1982), which gives the intensity of these lines relative to $I(\mathrm{H} \beta)$. Heydari-Malayeri \& Lecavelier des Etangs (1994) give an $I(\mathrm{H} \beta)$ intensity equal to $4.5 \times 10^{-11} \mathrm{erg} / \mathrm{s} / \mathrm{cm}^{2}$ for a region centered on the exciting stars of a radius equal to $21^{\prime \prime}$. In the same region we calculate the following ratios:

$$
\begin{aligned}
& \frac{I(\mathrm{SIV})}{I(\mathrm{H} \beta)}=0.34 \\
& \frac{I(\mathrm{NeIII})}{I(\mathrm{H} \beta)}=0.6 \\
& \frac{I(\mathrm{NeII})}{I(\mathrm{H} \beta)} \leq 0.34 .
\end{aligned}
$$

Assuming for N4 the physical parameters given in HeydariMalayeri \& Lecavelier des Etangs (1994 their Tables 6 and 8), i.e. $T_{\text {eff }} \sim 40000-45000 \mathrm{~K}, n \sim 100 \mathrm{~cm}^{-3},[\mathrm{~N}] /[\mathrm{H}]=11.3 \mathrm{e}-6$, $[\mathrm{O}] /[\mathrm{H}]=2.6 \mathrm{e}-4,[\mathrm{Ne}] /[\mathrm{H}]=3.2 \mathrm{e}-5,[\mathrm{~S}] /[\mathrm{H}]=6 \mathrm{e}-6$, and $\mathrm{a}$ metallicity equal to $Z / Z_{\text {sol }} \sim 0.5$, we found that the corresponding model from Stasińska (1982) (IDD2) predicts the following ratios:

$$
\begin{aligned}
& \frac{I(\mathrm{SIV})}{I(\mathrm{H} \beta)}=0.4 \\
& \frac{I(\mathrm{NeIII})}{I(\mathrm{H} \beta)}=0.6 \\
& \frac{I(\mathrm{NeII})}{I(\mathrm{H} \beta)}=0.05 .
\end{aligned}
$$

These values compare very well with those observed in N4, listed in Eq. (1). The model also predicts an HII region radius of $4.2 \mathrm{pc}$, which corresponds to an angular radius of 16.5" (assuming a distance for LMC equal to $52 \mathrm{kpc}$ ). This is comparable to the size of the NeIII/NeII emission if one does not include the extended emission on the south, outside the dust shell. Therefore, what we have called the HII region core, is justified here. It also roughly corresponds to the size of the aromatic-feature cavity.

We now compare the flux we obtained with previously published, similar data from ISO-SWS. Giveon et al. (2002) studied the mid-infrared fine structure lines emission of a sample of 
Table 2. Flux in the fine structure lines from ionized gas: first row gives the total fluxes; second row gives fluxes recovered in an aperture comparable to that of ISO-SWS.

\begin{tabular}{cccc}
\hline \hline Aperture & $F_{10.5 \mu \mathrm{m}(\text { SIV })}$ & $F_{12.8 \mu \mathrm{m}(\mathrm{NeII}+12.7)}$ & $F_{15.6 \mu \mathrm{m}}(\mathrm{NeIII})$ \\
$10^{-19} \mathrm{~W} / \mathrm{cm}^{2}$ & $10^{-19} \mathrm{~W} / \mathrm{cm}^{2}$ & $10^{-19} \mathrm{~W} / \mathrm{cm}^{2}$ \\
\hline Total & 68.7 & 51.6 & 65.0 \\
This paper in SWS aperture & 8.3 & 6.8 & 10.4 \\
Giveon et al. (2002) & 5.7 & 5.0 & 13.7 \\
\hline
\end{tabular}

galactic and extragalactic HII regions, based on ISO-SWS spectra. N4A belongs to this sample. In order to compare our flux with those given in Giveon et al. (2002), we should extract the flux in the exact same aperture as ISO-SWS $\left(14^{\prime \prime} \times 20^{\prime \prime}\right)$. This is not possible with the ISOCAM data because the pixel size of the images is 6" per pixel, and any rebinning is unwarranted for quantitative calculation because the pixel size undersamples the PSF at all ISOCAM wavelengths. Therefore we can extract the flux in a box that is as close as possible $\left(12^{\prime \prime} \times 18^{\prime \prime}\right)$ to the size of the ISO-SWS aperture centered on the HII region peak. The fluxes we recovered are listed in Table 2. They agree within $30 \%$ with the ISO-SWS fluxes. This is less than the total uncertainties, calculated taking into account the ISOCAM photometry uncertainties $(\simeq 10 \%)$, uncertainties introduced by the fitting that we estimate to be $\simeq 10 \%$, and the ISO-SWS photometric uncertainties $(\simeq 30 \%)$. Thus the total uncertainty is $>33 \%$ because we did not include in the calculation the uncertainties due to the differences in apertures and in pointings that cannot be estimated.

The agreement between the fluxes we recovered from the ISOCAM data treated with the continuum-plus feature-fitting technique and the ISO-SWS fluxes, makes us confident of the reliability of the analysis presented here. An interesting point is that the ISOCAM NeII+12.7AFE flux accounts for all the flux in the ISO-SWS spectrum for NeII alone. This suggests that the contribution of the $12.7 \mu \mathrm{m}$ dust emission in the center of the HII region is negligible, as it could have been guessed from the weakness of the other dust features. Therefore, in the HII region core, the NeIII/NeII value we calculated can be considered not as an upper limit but as the actual value that can now be directly compared with the typical ratios of poor metallicity systems given by Thornley et al. (2000).

\subsection{Dust emission}

\subsubsection{Comparison with the current dust model}

The AFE carriers are thought to be polycyclic aromatic hydrocarbons (PAHs), composed by 50 up to few a hundred atoms, and transiently heated mainly by far UV photons $(6-13.6 \mathrm{eV})$. Their emission intensity is proportional to the product of the dust density and the ISRF, although it certainly also depends on the hardness of the radiation field but in an unknown way. The VSGs emit through a mechanism that is intermediate between the stochastic heating typical of PAHs and the thermal equilibrium of the classical big grains. In quiescent regions dominated by the general interstellar field, the dominant VSGs' exciting mechanism is the stochastic heating that results in a smooth continuum peaking between 30-40 $\mu \mathrm{m}$. The emission mechanism becomes closer and closer to a standard thermal behavior when the radiation field gets more intense, such as in star-forming regions and PDRs. In these conditions the VSGs emission spectrum shifts toward shorter wavelengths, because of their quasi thermal behavior.
Our observations agree completely with the generally accepted picture to explain the dust feature and continuum emission at MIR wavelengths. First, we have shown in Sect. 3.2 that the morphology of the emissions in all dust features, including the NeII $+12.7 \mu \mathrm{m}$ if one excludes the HII region core, are very similar to one another, which strongly points towards a common origin and excitation mechanism for all AFEs.

Second, the spectra of N4 A presented in Fig. 2 can be fully explained with the classical picture illustrated above: in the HII region core the continuum longward $10 \mu \mathrm{m}$ increases simply because VSGs reach the thermal equilibrium regime and get hotter, thus producing a shift in their thermal emission spectrum to wavelengths shorter than $30 \mu \mathrm{m}$. The opposite happens in quiescent regions, such as the one corresponding to the eastern $\mathrm{CO}$ peak outside the HII region, where in fact almost no continuum is detected.

\subsubsection{Origin of the continuum at MIR wavelengths}

Since we have built "pure" continuum images at 6.75 and $15 \mu \mathrm{m}$, we can also investigate how these continua relate to AFEs. The origin of the MIR continuum in star-forming regions, PDRs and diffuse ISM, is a controversial issue. In principle it can be due to hot VSGs whose emission, as we have explained above, shifts into the MIR ISOCAM wavelengths and/or a continuum related to the AFE (maybe simply due to a superposition of unresolved and weak bands). Figure 10 shows the $6.7 \mu \mathrm{m}$ "pure" continuum's contours on the $15 \mu \mathrm{m}$ "pure" continuum emission image smoothed at the same resolution. Maps have been aligned on the WOH53 star. The two continua are very similar, thus suggesting a common origin. On the other hand, their morphology is not totally similar to the dust-feature emission morphology: they are more centrally peaked than the dust feature emission which forms a cavity. We therefore conclude that in N4, most of the continuum at MIR wavelengths arises from VSGs and not from unresolved weak aromatic bands, even at wavelengths shorter than $\sim 10 \mu \mathrm{m}$. This might not be the case in more quiescent regions like diffuse ISM, as indicated by the spectrum corresponding to the eastern $\mathrm{CO}$ peak (Fig. 2) where almost no continuum at all has been detected.

\subsubsection{Why is there no AFE in the HII region core?}

In this section we investigate the reason no AFEs are detected in the HII region core. The depression of AFE in high ISRF environments has been already observed in the Milky Way (Cesarsky et al. 1996b,a), Small Magellanic Cloud (Contursi et al. 2000) and in external galaxies (Galliano et al. 2003; Lu et al. 2003; Houck et al. 2004). Recently, a high-resolution study of the $3.3 \mu \mathrm{m}$ AFE (not included in the ISOCAM-CVF wavelength range) with ISAAC at the VLT in NGC253 and NGC1808 (Tacconi-German et al. 2005), has shown that AFE are strongly depressed with respect to the continuum close to where the super star clusters are located. For this reason these authors conclude 


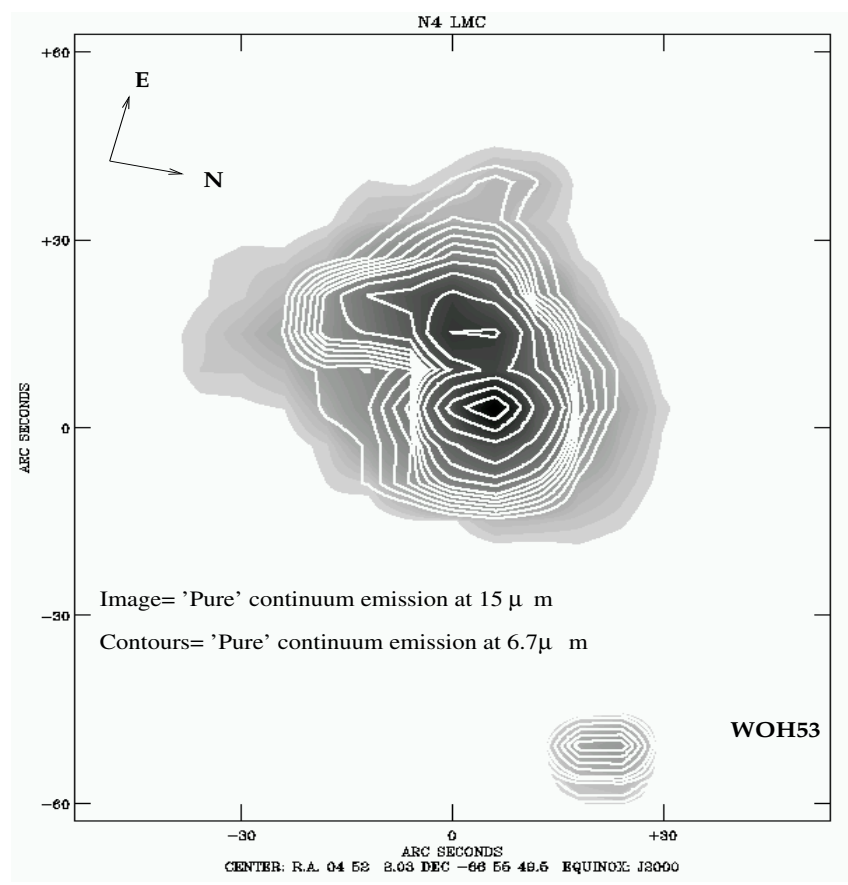

Fig. 10. Image: map from the $15 \mu \mathrm{m}$ "pure" continuum emission. Contours: "pure" continuum at $6.7 \mu \mathrm{m}$ smoothed to the $15 \mu \mathrm{m}$ resolution.

that the PAHs may be a better tracer of B stars rather than of more massive and earlier spectral type stars, as was already previously suggested by Peeters et al. (2004) on the basis of the analysis of a large sample of galactic HII regions observed with ISO.

In the case of N4, almost no AFE is detected at all in the HII region core. This can be explained in principle by three possibilities:

- 1) stellar winds are capable of evacuating the HII region from AFEs carriers but not from gas;

- 2) radiation pressure is capable of pushing the dust away but not the gas;

- 3) AFEs carriers are destroyed in very extreme radiation fields (RF).

Figure 3 shows that the "pure" continuum emission at $15 \mu \mathrm{m}$ has a secondary peak in the region where the ionized gas peaks. This is also visible in the MIR spectrum centered on SIV and the NeIII peak (Fig. 1 panel b) where almost no AFEs are visible but a significant continuum is still detectable. This shows that dust can survive in HII cores. As a consequence, the first two possibilities would imply that AFE carriers, VSGs, and gas are decoupled, which is physically quite improbable. We therefore conclude that the dominant mechanism has to be the destruction of AFE carriers. The question now is whether dust destruction is due to the intensity or the hardness of the radiation field, or both. Recent results from Wu et al. (2006) and Brandl et al. (2006) have shown that PAHs EW stay quite constant for a wide range of UV hardness as traced by the NeIII/NeII ratio and start to slightly decrease only at high $(\gtrsim 1) \mathrm{NeIII} / \mathrm{NeII}$ ratio in low-metallicity galaxies. More important is the result from $\mathrm{Wu}$ et al. (2006) who show that PAH EW is strongly anti correlated with the product of the radiation field intensity and hardness.

We tried to investigate this issue as illustrated in Fig. 11. Here both panels show the $7.7 \mu \mathrm{m}$ dust feature emission, the 6.75 , and $15 \mu \mathrm{m}$ "pure" continua emission as functions of the
ISRF energy density at $1600 \AA$ (left panel) and as functions of the NeIII/NeII ratio (right panel). The energy density at $1600 \AA$ is in units of the solar neighborhood at the same wavelengths as measured from Gondhalekar Phillips and Wilson (1980), and it has also been estimated in Contursi et al. (1998). These authors calculated the average emissions at each wavelength in annuli centered on the main ionizing stars till $3 \sigma$ level. The NeIII/NeII ratio is a tracer of the UV hardness. However, as already discussed in Sect. 4.1, at the ISOCAM spectral resolution, the NeII line emission at $12.8 \mu \mathrm{m}$ is blended with the AFE at $12.7 \mu \mathrm{m}$. Therefore, in the case of N4, this ratio is a lower limit everywhere but in the HII region core, where most of the flux can be ascribed to the NeII emission line alone, as shown in Sect. 4.1. In order to enhance differences among AFE and "pure" continua emission, we normalized each emission to its maxima.

In Fig. 11 we observe the following behavior:

- All dust components (AFEs, and the continua at the two wavelengths) show the same behavior: their emission increases until a certain value going from the outside toward the HII region center. They reach a maximum presumably on the PDR and then sharply decrease in the HII region core.

- The AFEs's drop is the steepest, followed by the the $6.7 \mu \mathrm{m}$ continuum and then by the $15 \mu \mathrm{m}$ continuum.

- The AFE's drop occurs first, i.e. at ISRF density and UV hardness lower than for the pure continua emission.

- The decline of all emission appears steeper when plotted versus the NeIII/NeII ratio than when plotted as function of the ISRF energy density.

These results definitely point to a scenario where, given the same conditions, AFE carriers are more easily destroyed than hot dust. In other words, these carriers are destroyed by less energetic UV photons than those necessary for destroying the carriers responsible for the "pure" continuum emission. If, following current dust models, these two types of carriers are associated with PAHs and VSGs, we can interpret this behavior as evidence that PAHs and VSGs are both destroyed but with different efficiencies. Moreover, assuming that the VSGs population has a certain size distribution, we can reasonably think that the continuum emission at shorter wavelengths is dominated by smaller particles than those responsible for the emission at longer wavelengths. In this framework, the drop of the continuum at $6.7 \mu \mathrm{m}$ is steepper than the drop of the $15 \mu \mathrm{m}$ continuum, can be interpreted as being the destruction mechanism more efficient on smaller molecules/grains. This means that: the destruction mechanism is likely to affect the dust size distribution.

This conclusion may have some important implications for understanding the ISM physics in dwarf galaxies. Thornley et al. (2000) find a NeIII/NeII ratio for IZw40 similar to what we measured in the very center of N4A (roughly a shell of $\sim 10 \mathrm{pc}$ in radius) but in a much larger region $(\sim 700 \times \sim 1000 \mathrm{pc})$. This means that the phenomena of destruction in such galaxies must occur in much larger regions than in normal galaxies. This can be explained by the fact that dwarf low-metallicity galaxies have an average UV spectrum that is harder than normal or starburst galaxies, due both to a higher star-formation rate per unit mass and to the presence of more young and massive star clusters. The UV photons can travel longer before being absorbed because these systems have less dust (Madden et al. 2006). All these factors point to a scenario where, in dwarf galaxies, PAHs have a higher probability of being destroyed than in normal metallicity environments, leading to an overall change of the galaxy dust size distribution. It also seems that, at least in not extremely poor 

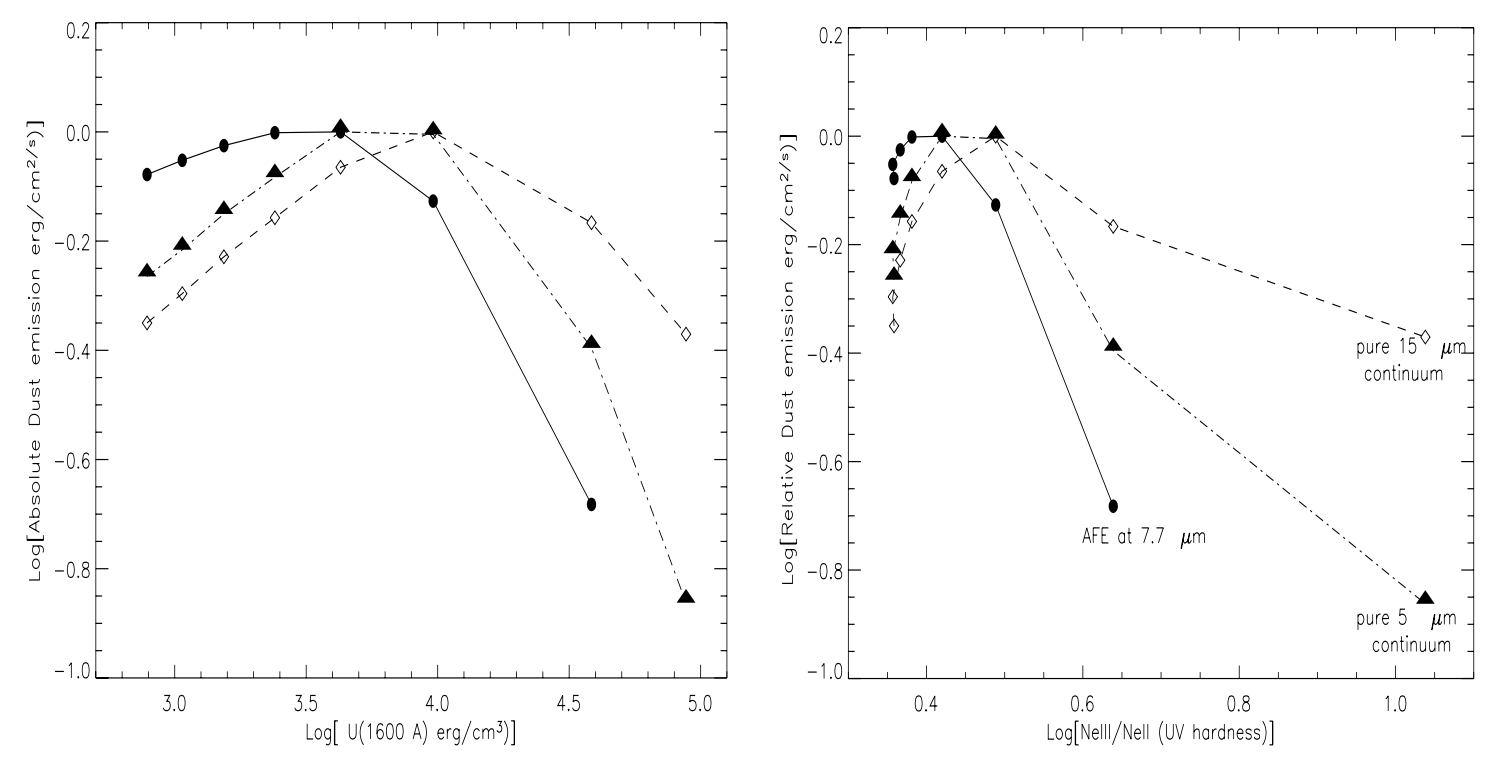

Fig. 11. The $7.7 \mu \mathrm{m}$ PAH emission (filled circle), $5 \mu \mathrm{m}$ and $15 \mu \mathrm{m}$ pure continuum emission (triangles and diamonds, respectively) versus the intensity of the radiation field at $1600 \AA$ (left panel) and NeIII/NeII ratio that traces the UV hardness (right panel). Values are above the $3 \sigma$ for the 3 wavelengths. In the right panel, the maximum at $7.7 \mu \mathrm{m}$ and $5 \mu \mathrm{m}$ have been scaled to the maxima at $15 \mu \mathrm{m}$ to ease the relative comparison among the different emissions.

metallicity systems, this phenomenon is more important than intrinsic modification of chemical dust properties.

Unfortunately, no firm conclusion can be achieved from this analysis about the possible different roles played by the ISRF intensity and hardness on the dust destruction mechanism. However, weak evidence that the latter has a stronger impact than the ISRF intensity might be deduced by the steeper drop that all dust emissions show with the NeIII/NeII ratio than with the ISRF energy density.

\subsection{Comparison with hot-dust properties in galactic environments.}

Metallicity is a parameter that may significantly affect dust. In low-metallicity environments, such as those expected in young distant galaxies, we expect dust to have intrinsic chemicalphysical properties that are very different from those observed in normal metallicity galaxies, just because dust forms and grows in an ISM with very different chemical enrichment and mechanical inputs (from both stellar winds and supernovae). These might lead to not only overall under-abundance of carbon-based grains with respect to the abundance in normal metallicity environments but also to dust feature emission profiles, widths, and intensity ratios different from those found in normal-metallicity ISM.

In Sect. 4.2.3 we have shown that dust destruction in N4 could be, at least in part, responsible for the lack of small molecules/grains, already detected on a much larger scale with IRAS in the Magellanic Clouds (Sauvage et al. 1990), although we might have a lower total amount of carbon-based molecule/grains than in Galactic HII regions. On the other hand, we detected strong aromatic features that are very similar to what is observed in the Milky Way's ISM. In this section we analyse the N4 dust features widths and intensity ratios in more detail, in order to understand their physical characteristics and how they compare with similar observations in galactic environments.

\section{- Dust feature intensities}

Figure 12 shows a comparison between the PDR spectrum in N4 (panel c in Fig. 2) and the spectrum of one pixel in the PDR of NGC7023, after having subtracted a straight line continuum from both and scaled the spectrum of NGC7023 up to match the $7.7 \mu \mathrm{m}$ feature of $\mathrm{N} 4$ by eye. There is no evidence, at least at the CVF spectral resolution $(\lambda / \Delta \lambda \sim 50)$, of grain/molecules modifications due to metallicity resulting in differences in the features' profiles or peak-wavelength shifts. Indeed, the shape and the relative intensities of the $6.2,7.7$, and $8.6 \mu \mathrm{m}$ features in NGC7023 and N4 almost match perfectly. There is an indication of a relatively lower $11.3 \mu \mathrm{m}$ feature in N4 than in NGC7023, which may be due to a higher level of PAH ionization in $\mathrm{N} 4$. This is plausible, since N4 is more active than NGC7023, even in its PDR region, as shown by the presence of ionized gas lines that are totally absent in the NGC7023 spectrum (but see more details below in this section). This confirms the result obtained by Vermeij et al. (2002), who find that the AFEs characteristics of LMC HII regions are similar to those in the Milky Way.

\section{- Dust feature widths}

The analysis of the dust features conducted in this work is based on the assumption that their profiles are represented by a Lorentzian. In this context, the widths of the features have a physical explanation. Boulanger et al. (1998) proposed that the emitting mechanisms of the AFE's carriers is the internal vibrational redistribution. The bands widths arise from intramolecular broadening processes in large molecules and are related to the very short life-time of the emitting levels. One of the byproducts of the fitting technique we used are the widths of the fitted Lorentzian through the entire data cube (i.e. wavelengths). We can therefore compare what was obtained for N4 with the range of widths that Boulanger et al. (1998) found for typical galactic regions (namely $\rho$ Ophiuchus and NGC7023). Figure 13 shows this comparison. We calculated the mean widths of N4 obtained by the fit, taking into account all pixels with a signal greater than 


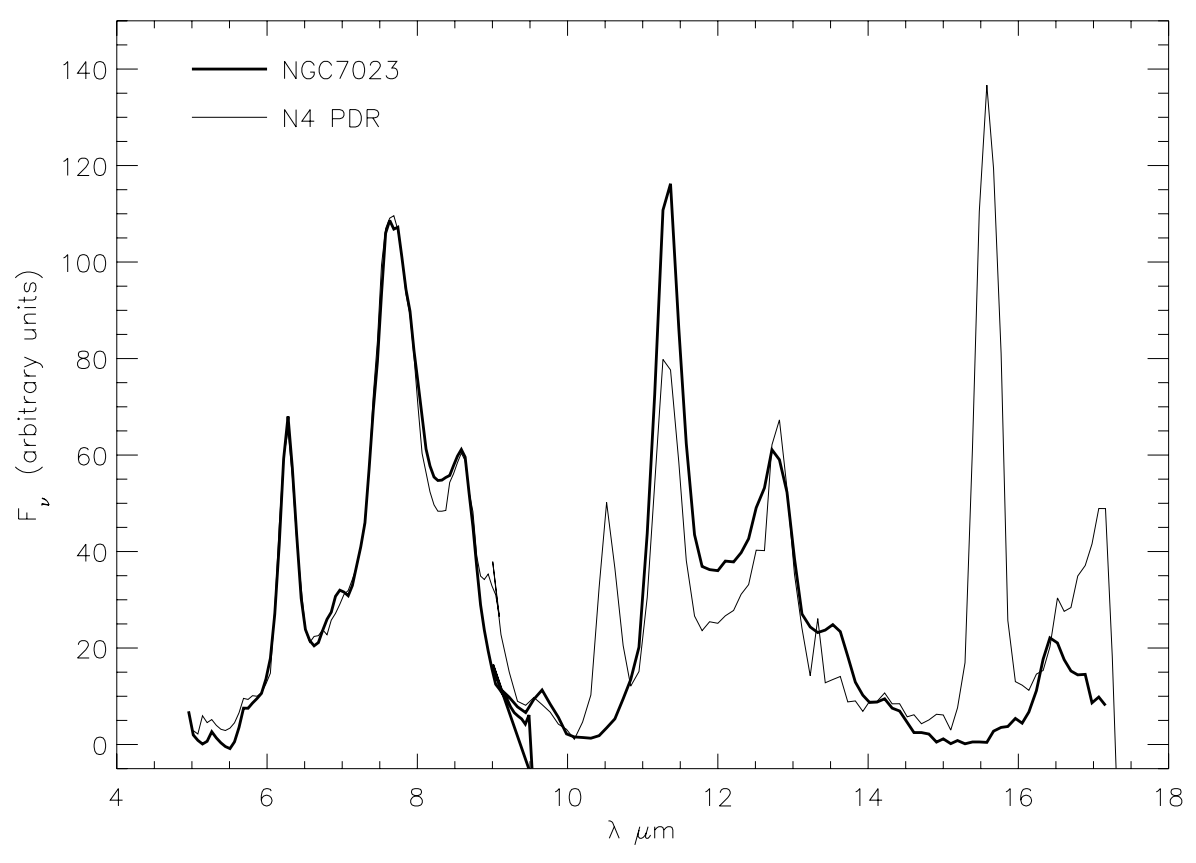

Fig. 12. Comparison between the spectrum of the galactic region NGC7023 (thick solid line) and the PDR spectrum of N4 (thin solid line), after being continuum-subtracted. NGC7023 spectrum has been scaled to match the $7.7 \mu \mathrm{m}$ feature of $\mathrm{N} 4$. the $3 \sigma$ of the rms at each wavelength (filled circles) and the mean only for the PDR region (filled triangles), i.e. the same region shown in panel c of Fig. 2. The open stars corresponds to the mean FWHM values found for the two galactic regions by Boulanger et al. (1998). We exclude the $12.7 \mu \mathrm{m}$ feature because we fitted it with a Gaussian and not with the Lorentzian as in Boulanger et al. (1998). The agreement is very good at all wavelengths showing that this parameter is also very similar to what is observed in galactic environments despite the fact that $\rho$ Ophiuchus and NGC7023 are in ISRF much less intense than N4.

\section{- Dust feature ratios}

Reach et al. (2000) find that the $11.3 / 7.7 \mu \mathrm{m}$ ratio is higher in SMCB1-1 than in various ISM regions in the Milky Way. SMCB1-1 ia a relatively quiescent cloud in the Small Magellanic Cloud (SMC) that is not associated with ongoing star formation. This difference was attributed to an enhancement of the $\mathrm{C}-\mathrm{H}$ bonds with respect to the $\mathrm{C}-\mathrm{C}$ bonds, due to the higher $\mathrm{H} / \mathrm{C}$ abundance ratio in the SMC than in the Milky Way.

We have produced AFE feature ratio maps (not shown here), to study their variation across the region. The mean $11.3 / 7.7 \mu \mathrm{m}(\mathrm{C}-\mathrm{H} / \mathrm{C}-\mathrm{C})$ value is 0.25 very similar to what was found by Boulanger et. al. (1996) in $\rho$-Oph and lower than the value in SMCB1-1 (0.8). A collection of $11.3 / 7.7 \mu \mathrm{m}$ ratios in different galactic ISM by $\mathrm{Lu}$ et al. (1998) though, clearly indicates that this ratio has a large dispersion even in similar ISM phases. Therefore, the analysis of the dust-feature ratios confirms that the dust feature ratio in $\mathrm{N} 4$ are comparable to what is found in many other regions. An important result is that this ratio is quite constant on the dust shell, indicating that whatever the state of the AFE carriers, i.e. ionized/neutral, hydrogenated or modified from metallicity, there are no strong differential effects on the dust feature emission and, therefore presumably, on the dust conditions, at least at this spatial resolution ( $\sim 2$ pc assuming a LMC distance of $52 \mathrm{kpc}$ ).

The changes in the profiles of the dust features and their relative intensities can tell a lot about the physical state of AFEs carriers if associated with PAHs (Pauzat et al. 1997;
Le Page et al. 2003; Dartois \& D'Hendecourt 1997; Bakes et al. 2001). While the first analysis is impossible at the ISOCAM-CVF spectral resolution, we can analyze how AFE ratios change in N4 as a function of the ISRF intensity.

It is believed that PAHs in PDRs are mostly ionized. Ionization of PAHs leads to an enhancement of the intensities of the features arising from the $\mathrm{C}-\mathrm{C}$ bonds $(6.2$ and $7.7 \mu \mathrm{m}$ bands in the ISOCAM wavelengths range) with respect to those arising from the $\mathrm{C}-\mathrm{H}$ bonds $(8.6 \mu \mathrm{m}$ in-plane and outof plane (oop) solo, duo, trio, and quatro at 11.3, 11.9, 12.7, and $14 \mu \mathrm{m}$, respectively). Dehydrogenation weakens the $\mathrm{C}-\mathrm{H}$ bonds. Therefore the ratio between any feature arising from $\mathrm{C}-\mathrm{H}$ bonds with a feature arising from $\mathrm{C}-\mathrm{C}$ bonds, should decrease as ionization and dehydrogenation increase. Figure 14 upper panel shows the $11.3 / 7.7 \mu \mathrm{m}$ ratio (oop C-H/ vibration $\mathrm{C}-\mathrm{C}$ ) as function of the ISRF. There is a weak decreasing trend indicating that either ionization or dehydrogenation are perhaps weakly increasing when approaching the HII region core. On the other hand, there is an increase of this ratio in the very center probably due to the fact that here smaller molecules (which emit at shorter wavelengths) are destroyed before the larger species. Verstraete et al. (1996) analyzed the oop $\mathrm{C}-\mathrm{H}$ to in-plane $\mathrm{C}-\mathrm{C}$ dust-feature ratio, traced by the $(11-13) /(6.2+7.7) \mu \mathrm{m}$ ratio. Theoretical predictions show that this ratio is 2.5 for neutral PAHs and 0.25 for cations. The bottom panel of Fig. 14 shows a similar ratio where, in place of the 11-13 emission features, we calculated the $11.3+12.8 \mu \mathrm{m}$ emission. These ratios are well below those predicted for neutral PAHs everywhere in N4, and very close to the value for ionized PAHs, suggesting that PAHs are ionized in the whole region. Note that in the HII region core, this ratio is an upper limit because the $12.7 \mu \mathrm{m}$ emission is almost entirely due to NeII rather than the AFE at $12.7 \mu \mathrm{m}$. We therefore conclude that, if PAHs are the carriers responsible for the dust features, they are almost totally ionized in N4, and that their state of ionization and/or dehydrogenation is constant all over the region. In order to detect the transition between neutral to ionized $\mathrm{PAH}$, one probably has to trace the PAH emission from diffuse ISM to active regions. 


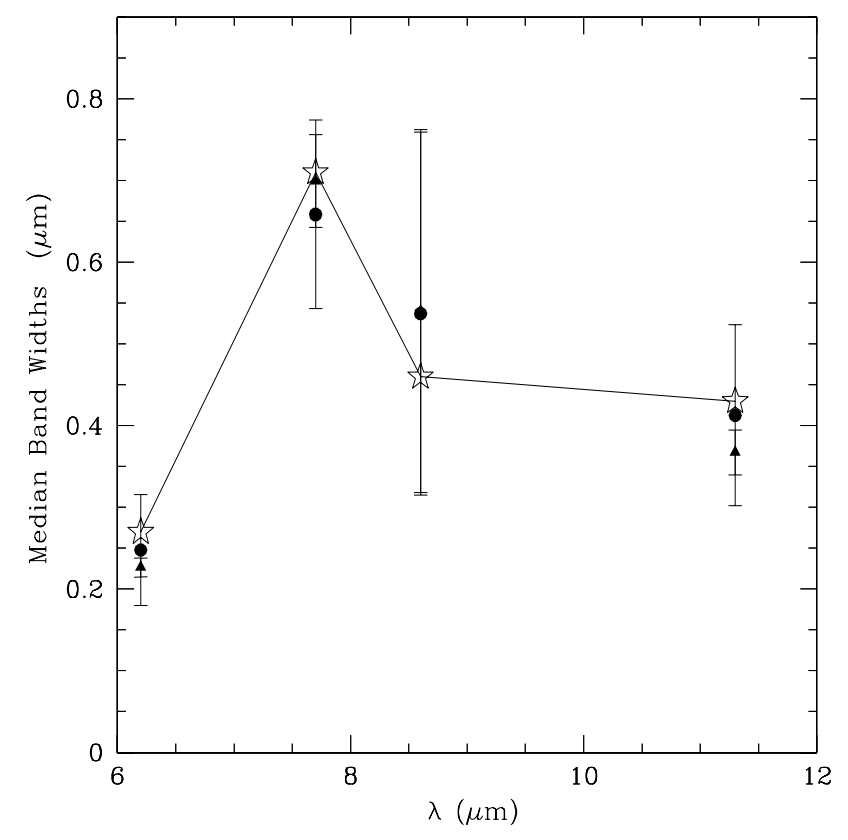

Fig. 13. Typical widths of the AFEs obtained by the Lorentzian fit for all N4 (filled circles), the PDR only (solid triangles), and the typical values found with the same fitting technique by Boulanger et al. (1998) in $\rho$ Oph and NGC7023 (open stars).

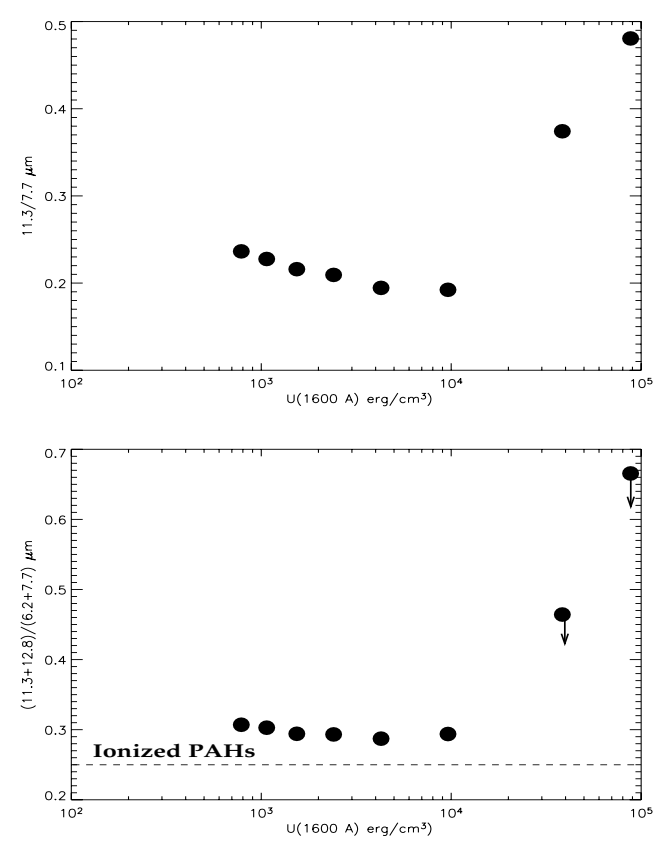

Fig. 14. Different dust feature ratios as functions of the ISRF. Upper panel: the 11.3/7.7 $\mu \mathrm{m}$ dust feature ratio, which traces the [out of plane $\mathrm{C}-\mathrm{H}] /[$ vibration C-C] ratio. Bottom panel: the $(11-13) /(6.2+7.7) \mu \mathrm{m}$ dust feature ratio, which traces the [out of plane $\mathrm{C}-\mathrm{H}] /[$ in plane $\mathrm{C}-\mathrm{C}$ ] dust features ratio.

In conclusion, we have shown that three fundamental parameters of AFE, namely, emission intensities, features typical widths and emission ratios, are all very similar to what is typically found in normal-metallicity ISM.

\section{The stellar content}

The NIR data presented in this paper unveil at least 7 bright sources. Figure 15 shows a NIR color-magnitude diagram (right panel) and a $J H K_{s}$ color-color (left panel) of stars numbered as in Fig. 5 whose photometry is reported in Table 1. These are shown as filled pentagons in Fig. 15. This figure includes for comparison the IR data obtained for two other LMC star forming regions, 30 Doradus (Rubio et al. 1998) and N11 (Barbá et al. 2003). In each figure's panel the zero age main sequence (ZAMS) is plotted asadopted from Hanson et al. (1997), as well as the extinction tracks for the reddening law of Rieke \& Lebofsky (1985).

Note that the extinction for star \#4 is likely to be much higher than the average, as this star falls in the dust shell and on the $15 \mu \mathrm{m}$ continuum peak. Four N4 IR sources show colors that are consistent with reddened main sequence $\mathrm{O}$ stars, while the other three are IR objects.

From Fig. 15 we can conclude the following:

1) Stars \#1 and \#3 are probably moderately reddened mainsequence $\mathrm{O}$ stars with $A_{\mathrm{V}} \sim 3$. They are the main ionizing stars responsible for the HII region (Heydari-Malayeri \& Lecavelier des Etangs 1994) and labeled as A and B in Fig. 1.

2) Stars \#5 and \#6 are highly reddened main-sequence $O$ stars with $A_{V}>10$. They do not have optical counterparts.

3) The IR objects labeled \#4, \#5, and \#2 have large IR excess and their colors are not that of reddened young mainsequence stars. They lie on the border of the molecular cloud seen in CO (Contursi et al. 1998) and at the edge of the cavity formed by the ionizing stars. These three objects have $J$ band brightness corresponding to Class I and /or Herbig Ae/Beobject candidates (see Brandner et al. 2001). Stars \#7 and \#4 have the largest IR excess of the sample. They have the IR characteristics of massive young stellar objects (YSOs) similar to N122 in 30Dor. Object \#2 is an extended object that shows nebulosity around and a second red component.

\subsection{The nature of star \# 4}

This is the brightest member of a small cluster that is of particular interest because it corresponds to a bright point-source emission in the hot dust pure continuum emission. Its position on the NIR color-color and color-magnitude diagram is similar to that of IR sources found in 30 Doradus, i.e. source W30, N122, and N126 (Rubio et al. 1998). These 30 Doradus sources were resolved into multiple, compact, young massive systems from high-resolution HST/NICMOS observations, with a dominant IR source (Walborn et al. 2002; Walborn et al. 1999). The IR color of star \#4 can be either interpreted as an early $\mathrm{O}$ type star with $A_{V}=20$ and with an IR K excess of about 4 magnitudes or a multiple object containing several ZAMS O3 stars extinguished by $20 \mathrm{mag}$. In fact, the star profile is wider than the point-spread function derived for the stars in the $K_{\mathrm{s}}$ image, which could be a hint of the presence of a much redder component, thus favoring the hypothesis of a multiple system.

We can check the results on the nature of star \#4 obtained from NIR photometry, by comparing of our ISOCAM and NIR data with those published by the ISOGAL team (Felli et al. 2002). The LW2 and LW3 broad band magnitudes obtained through PSF fitting are: $m(\mathrm{LW} 2)=6.1$ and $m(\mathrm{LW} 3)=3.4$. Then, the position of star \#4 on the [15]-[7]-[15] magnitude-color diagram published by Felli et al. (2002) falls exactly in the region occupied by YSOs.

A third indication that this object indeed contains a YSO, is given by its CVF spectrum shown in Fig. 16. It shows a deep silicate absorption at $\sim 10 \mu \mathrm{m}$ that suggests this is a highly embedded source. This is also confirmed by the fact that star \#4 

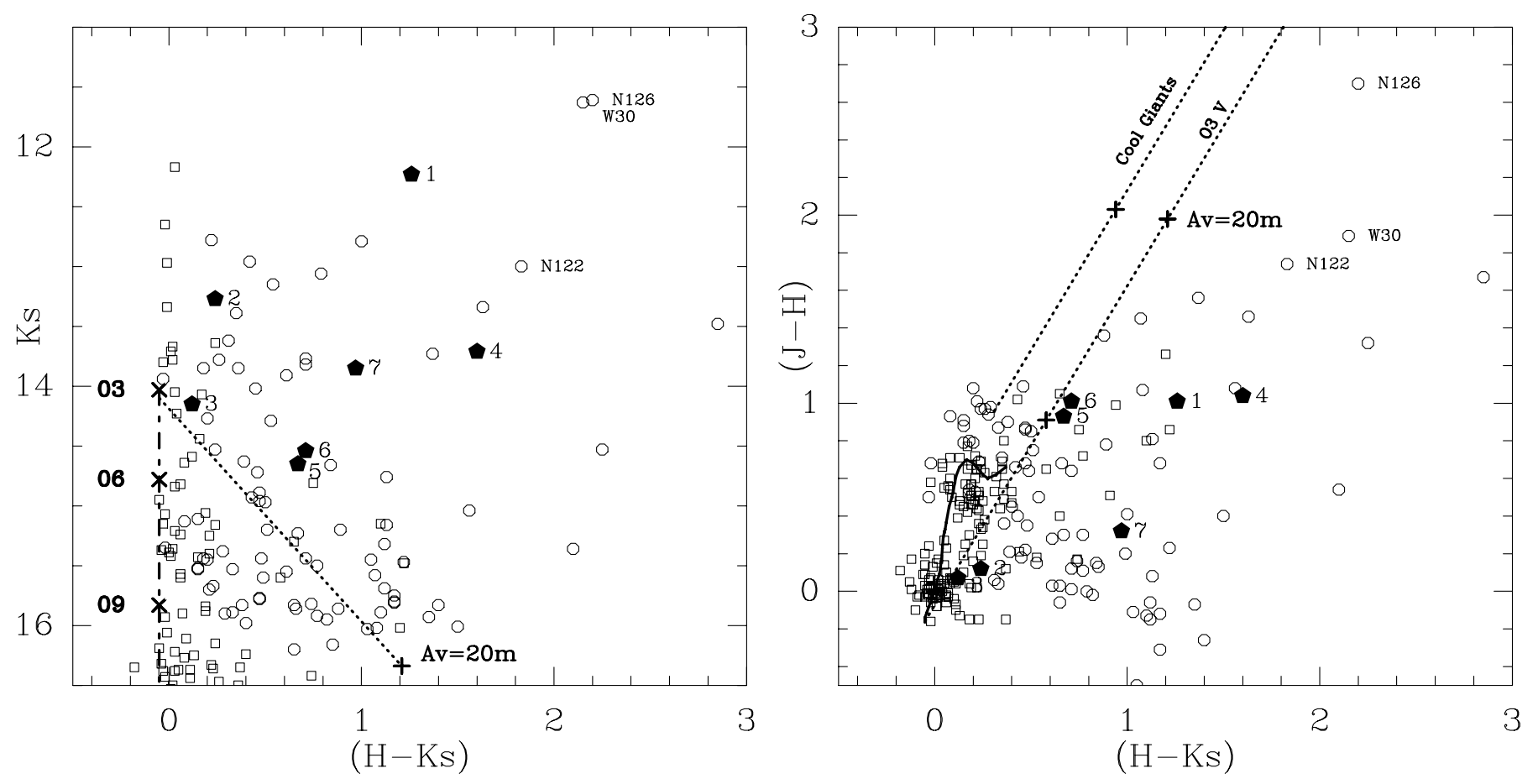

Fig. 15. NIR color-magnitude (left) and color-color (right) diagrams of $\mathrm{N} 4$ with the IR sources shown as red filled points. For comparison we include the 30 Doradus IR sources as open circles and the N11 IR sources as open squares. In the color-magnitude diagram the upper zero-age main sequence between $\mathrm{O} 3 \mathrm{~V}$ and $\mathrm{O} 9 \mathrm{~V}$ corresponds to a distance modulus of 18.6 and is indicated by a dashed line. The reddening track for a normal O3 V star is plotted with a dotted line and extends to $A_{\mathrm{V}}=20 \mathrm{mag}$. In the color-color diagram, the main-sequence locus from O3 V to M2 V and the cool-giant branch are indicated by a solid line. The reddening tracks for normal $\mathrm{O} 3 \mathrm{~V}$ and cool giant stars are plotted as dashed lines, with crosses indicating $A_{\mathrm{V}}=10$ and 20 mag. Sources 1, 4, and 7 show IR excesses that cannot be explained by reddening.

corresponds to the strongest peak of the $15 \mu \mathrm{m}$ pure-continuum emission. In the spectrum two weak absorption features are also visible at $\sim 13 \mu \mathrm{m} \sim 15 \mu \mathrm{m}$. We checked whether they are due to memory effects of the detector after a glitch. The result is that, most likely, the feature at $13 \mu \mathrm{m}$ is fake but that at $15 \mu \mathrm{m}$ seems real. If this is so, it could be associated with the solid $\mathrm{CO}_{2}$ bending mode at $15.2 \mu \mathrm{m}$ or to gas-phase $\mathrm{CO}_{2}$ at $15.0 \mu \mathrm{m}$. Both types of emission are detected in YSOs (van Dishoeck 2004). The fact that the overall spectrum shown in Fig. 16 does not look like a typical YSO MIR spectrum (van Dishoeck 2004) could be due to the ISO spatial resolution; the ISOCAM beam is in fact sufficiently large ( $2 \mathrm{pc}$ at the LMC distance) to include a considerable amount of ISM surrounding the YSO, as suggested by the presence of AFEs. Since this feature, together with the solid $\mathrm{CO}_{2}$ stretch mode at $4.25 \mu \mathrm{m}$ and $\mathrm{H}_{2} \mathrm{O}$ at $3 \mu \mathrm{m}$ (out of our wavelength range), has been observed at a higher resolution with ISO-SWS in some ultra compact HII regions (Roelfsema et al. 1998), this would support our hypothesis that star \#4 is an embedded YSO.

\section{Conclusion}

In this paper we have analyzed the ISOCAM CVF and new $J H K_{\mathrm{s}}$ photometry data of the HII region complex N4 in the Large Magellanic Clouds. The analysis had two principal aims: 1) to relate dust, gas and stellar content; 2) to look for effects of the lower-than-galactic metallicity on the dust features. The main conclusions are the following.

- The overall MIR spectral characteristics of N4 can be fully explained with the current dust models. The properties (continuum + dust features + gas lines) are those of a typical galactic HII complex which can be interpreted as the

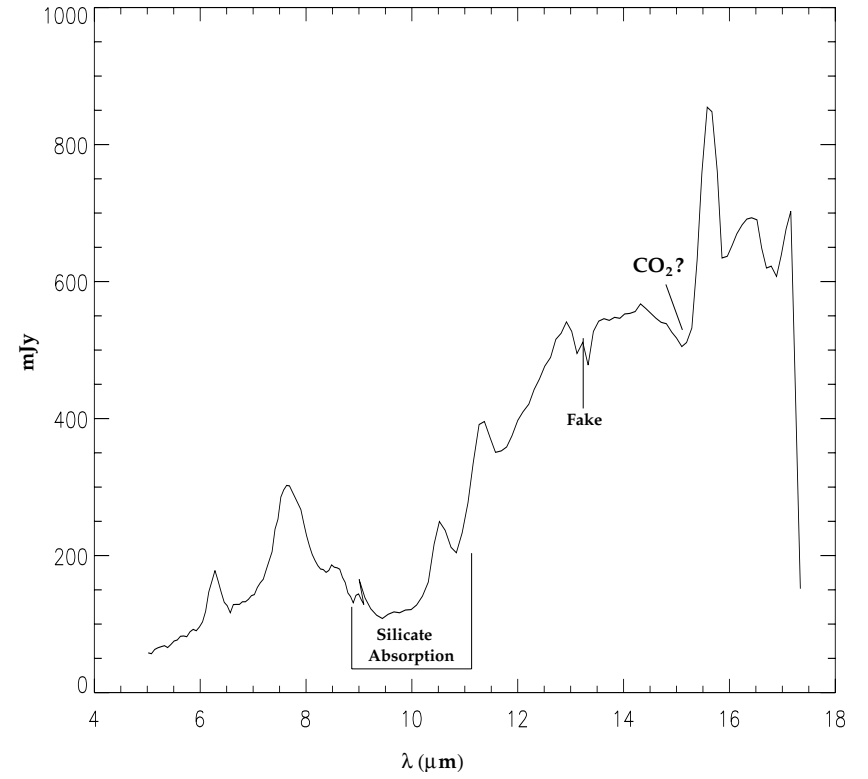

Fig. 16. ISOCAM CVF spectrum of star \#4 corresponding to the $15 \mu \mathrm{m}$ pure continuum peak.

combination of three primary components: HII region core+ PDR+ parental molecular cloud.

- Thanks to a fitting technique through the entire CVF data cube, we can produce maps in the single dust features, gas lines, and pure continuum. We find that the HII region core is completely devoid of dust features. This dust cavity contains most of the ionized gas. The pure continuum emission seems 
to have the same origin at all wavelengths, but it probably arises from a population of grains different from the AFE carries. Following the current dust models, this component can be totally ascribed to VSGs.

- The comparison between the ISM emission and the stellar content of N4 derived from NIR photometric data reveals that the two bluest stars are the main ionizing stars of N4A: they reside in the HII region core, i.e. in the center of the AFE cavity and where the ionized gas peaks. The pure continuum emission peaks on a point source that shows strong evidences of being a group of massive young stars containing a deeply embedded YSOs.

- A detailed comparison of the relative spatial distribution and intensities of the dust features as a function of the intensity of the radiation field suggests that the HII region core does not contain PAHs because they are destroyed. We show that this mechanism is selective depending on the grain sizes, with the smallest grains/big-molecules being destroyed first. This can lead to an overall modification of the grain-size distribution, which can be significant in dwarf galaxies of relatively low metallicity, where these processes act on large portions of their ISM. We also argue that this mechanism is more important than any intrinsic dust modification due to the relatively low metallicity of LMC.

- The comparison between the widths and the intensity ratios of the dust features in N4 and in other galactic environments shows that there are no evident and significant signs of metallicity effects on AFE's carriers in N4

- Analysis of the dust features intensities ratios and their comparison with model predictions indicate that, if PAHs are the particles responsible for the AFEs, they are fully ionized over the entire analyzed region.

- The IR stellar content of the N4 shows several bright IR sources with characteristics of massive, early $\mathrm{O}$ type stars similar to those found in 30Doradus. The IR spectroscopy of these sources would confirm their very young and massive nature.

\section{References}

Bakes, E. L. O., Tielens, A. G. G. M., \& Bauschlicher, C. W. 2001, ApJ, 556, 501

Barbá, R., Rubio, M., Roth, M., et al. 2003, AJ, 125, 1940

Boulanger, F., Boisssel, P., Cesarsky, D., \& Ryter, C. 1998, A\&A, 339, 194

Boulanger, F., Lorente, R., Miville Deschenes, M. A., et al. 2005, A\&A, 436, 1151

Bradner, W., Grebel, E. K., Barbá, R. et al. 2001, AJ, 122, 858

Brandl, B. R., Bernard-Salas, J., Spoon, H. W. W., et al. 2006, ApJ, 653, 1129

Cesarsky, D., Lequeux, J., Abergel, A., et al. 1996a, A\&A, 315, 305

Cesarsky, D., Lequeux, J., Abergel, A. et al. 1996b,A\&A, 315, 309

Contursi, A., Lequeux, J., Hanus, M., et al. 1998, A\&A, 336, 662

Contursi, A., Lequeux, J., Cesarsky, D., et al. 2000, A\&A, 362, 310

Dartois, E., \& D’Hendecourt, L. 1997, A\&A, 323, 534

Ferland, G. J., Korista, K. T., Verner, D. A., et al. 1998, PASP, 110, 761

Galliano, F., Madden, S. C., Jones, A. P., et al. 2003, A\&A, 407,159

Giveon, U., Sternberg, A., Lutz, D., et al. 2002, ApJ, 566, 880

Gondhalekar, P. M., Phillips, A. P., \& Wilson, R. 1980, A\&A, 85, 272

Hanson, M. M., Howard, I. D., Conti, P. S. 1997, ApJ, 489, 698

Henize, K. G. 1956, ApJSS, 2, 315

Heydari-Malayeri, M., \& Lecavelier des Etangs, A. 1994, A\&A, 291, 960

Houck, J., Charmandaris, V., Brandl, B., et al. 2004, ApJSSI

Felli, M., Testi, L., Shchuller, F., et al. 2002, A\&A9392,971

Le Page, V., Snow, T. P., \& Bierbaum, V. M. 2003, ApJ, 584, 316

Peeters, E., Spoon, H. W. W., \& Tielens, A. G. G. M. 2004, ApJ, 613, 986

Lu, N., Helou, G., Werner, M. W., et al. 2003, ApJ, 588, 199

Madden, S. C., Galliano, F., Jones, A. P., et al. 2006, A\&A, 446, 877

Pauzat, F., Talbi, D., \& Ellinger, Y. 1997, A\&A, 319,318

Rieke, G. H., \& Lebofsky, M. J. 1985, ApJ, 228, 618

Roelfsema, P., Cox, P., Hessler, M. F., et al., ed. J. L. Yum, \& R. Liseau, ASP conf. Ser., 132

Rubio, M., Barbá, R., Walborn, et al. 1998, AJ, 116, 1708

Sauvage, M., Vigroux, L., \& Thuan, Y. X. 1990, A\&A, 237, 296

Stasińska, G. 1982, A\&AS, 48, 2999

Strom, K. M., Kepner, J., \& Strom, S. E. 1995, ApJ, 438, 829

Tacconi-Garman, L. E., Sturm, E., Lehnert, M., et al. 2005, A\&A, 432, 91

Thornley, A. D., Förster Schreiber, N. M., Lutz, D., et al. 2000, ApJ, 539, 641 Van Dishoeck, E. F. 2004, ARA\&A, 42, 119

Vermeij, R., Peeters, E., Tielens, A. G. G. M, et al. 2002, A\&A, 382, 1042

Zavagno, A., Deharveng, L., Comerón, F., et al. 2006, A\&A, 446, 171

Verstraete, L., Puget, J. L., Falgarone, E. et al. 1996, A\&A, 315, L337

Walborn, N. R., Barbá, R., Brandner, W. 1999, AJ, 117, 225

Walborn, N. R., Maiz-Apellaniz, J, Barbá, R. 2002, AJ, 124, 1601

Wu, Y., Charmandaris, V., Hao, L., et al. 2006, ApJ, 639, 157 